\title{
Competitive exclusion in a two-species chemotaxis model
}

\author{
C. Stinner · J. I. Tello $\cdot$ M. Winkler
}

\begin{abstract}
We consider a mathematical model for the spatio-temporal evolution of two biological species in a competitive situation. Besides diffusing, both species move toward higher concentrations of a chemical substance which is produced by themselves. The resulting system consists of two parabolic equations with Lotka-Volterratype kinetic terms and chemotactic cross-diffusion, along with an elliptic equation describing the behavior of the chemical. We study the question in how far the phenomenon of competitive exclusion occurs in such a context. We identify parameter regimes for which indeed one of the species dies out asymptotically, whereas the other reaches its carrying capacity in the large time limit.
\end{abstract}

\section{Introduction}

We consider two biological species which compete for the resources and migrate towards a higher concentration of a chemical produced by themselves. Here the movement of the two populations is governed by diffusion and chemotaxis. We further

C. Stinner - M. Winkler

Institut für Mathematik, Universität Paderborn, 33098 Paderborn, Germany

J. I. Tello (凶)

Matemática Aplicada, EU Informática, Universidad Politécnica de Madrid, 28031 Madrid, Spain

e-mail: jtello@eui.upm.es 
assume that the populations proliferate, that the mutual competition between them takes place according to the classical Lotka-Volterra dynamics and that the chemical signal diffuses much faster than the two populations. Denoting the population densities by $u(x, t)$ and $v(x, t)$ and the concentration of the chemoattractant by $w(x, t)$, classical models (see Murray 1993) lead to the system

$$
\begin{cases}u_{t}=d_{1} \Delta u-\chi_{1} \nabla \cdot(u \nabla w)+\mu_{1} u\left(1-u-a_{1} v\right), & x \in \Omega, t>0, \\ v_{t}=d_{2} \Delta v-\chi_{2} \nabla \cdot(v \nabla w)+\mu_{2} v\left(1-v-a_{2} u\right), & x \in \Omega, t>0, \\ -\Delta w+\lambda w=k u+v, & x \in \Omega, t>0,\end{cases}
$$

under homogeneous Neumann boundary conditions

$$
\frac{\partial u}{\partial v}=\frac{\partial v}{\partial v}=\frac{\partial w}{\partial v}=0, \quad x \in \partial \Omega, t>0,
$$

and initial conditions

$$
u(x, 0)=u_{0}(x), \quad v(x, 0)=v_{0}(x), \quad x \in \Omega,
$$

in a bounded domain $\Omega \subset \mathbb{R}^{n}, n \geq 1$, with smooth boundary, where $d_{i}, \chi_{i}, \mu_{i}, a_{i}(i=$ $1,2), \lambda$ and $k$ are positive parameters.

In order to describe the competition of two species, the associated Lotka-Volterra ODE system

$$
\begin{cases}u^{\prime}=\mu_{1} u\left(1-u-a_{1} v\right), & t>0, \\ v^{\prime}=\mu_{2} v\left(1-v-a_{2} u\right), & t>0,\end{cases}
$$

has been studied extensively. It is well-known that if

$$
a_{1}>1>a_{2} \geq 0
$$

and both species are initially positive then the second population outcompetes the first in the sense that $u(t) \rightarrow 0$ and $v(t) \rightarrow 1$ as $t \rightarrow \infty$. A proof of this result and of extensions to systems with more populations is given in (Zeeman 1995, Theorem 2.1). It is the objective of the present work to investigate in how far this phenomenon, usually referred to as competitive exclusion, can be observed also in cases when both species move towards increasing concentrations of a signal which they produce themselves.

The influence of chemotaxis on the dynamics of biological species competing for resources like nutrients or space is for instance pointed out in Celani and Vergassola (2010), Hawkins (2011), Hibbing (2010) and Tindall (2008). Particular fields of relevance include economically important situations when different bacteria interact with crop plants, where beyond standard kinetics, the respective overall competitive fitnesses are crucially affected by chemotaxis and motility, see Brencic and Winans (2005), Vande Broek and Vanderleyden (1995) and Yao and Allen (2006). Derivations of related mathematical models can be found in Kelly et al. (1988), Lauffenburger 
(1991), Painter and Sherratt (2003) and some basic mathematical aspects such as the global existence of solutions to models which involve both chemotaxis and competition are addressed in Kuiper (2001), Zhang (2006). Moreover, for some particular models the existence and stability of steady states reflecting either competitive exclusion or coexistence have already been studied analytically, see Dung (2000), Dung and Smith (1999), Wang and $\mathrm{Wu}$ (2002) and Zhang (2006); however, to the best of our knowledge the literature does not provide any qualitative information on the solution behavior in the context of competitive exclusion when chemotaxis as well as competitive terms involving both species are present.

Concerning the problem considered in this paper, in case of $a_{1}, a_{2} \in[0,1)$ it has been shown in Tello and Winkler (2012) that (1.1)-(1.3) possesses a unique positive steady state and conditions on the parameters $\mu_{i}$ and $\chi_{i}$ are established which ensure its global asymptotic stability. In contrast to this result of coexistence of the species we shall show here that in presence of (1.4) competitive exclusion will take place, provided that the influence of chemotaxis is sufficiently small.

In order to state our results in this direction, let us introduce the ratios

$$
q_{1}:=\frac{\chi_{1}}{\mu_{1}} \text { and } q_{2}:=\frac{\chi_{2}}{\mu_{2}} .
$$

It turns out that in our analysis, besides the number $k$ these parameters will play the role of key parameters with regard to the effect in question. In particular, we shall see that if both $q_{1}$ and $q_{2}$ are sufficiently small then competitive exclusion occurs for any solution $(u, v, w)$ with $v \not \equiv 0$.

More precisely, in addition to (1.4) our overall assumptions are

$$
\begin{aligned}
& k, q_{1} \text { and } q_{2} \text { are nonnegative and such that } q_{1} \leq a_{1}, q_{2}<\frac{1}{2} \text { and } \\
& k q_{1}+\max \left\{q_{2}, \frac{a_{2}-a_{2} q_{2}}{1-2 q_{2}}, \frac{k q_{2}-a_{2} q_{2}}{1-2 q_{2}}\right\}<1 .
\end{aligned}
$$

Observe that these can be rewritten in separate conditions for $k, q_{2}$ and $q_{1}$ in such a way that we require

$$
\begin{aligned}
& k \geq 0, \\
& q_{2} \in\left[0, \frac{1}{2}\right) \text { is such that } q_{2}< \begin{cases}\frac{1-a_{2}}{2-a_{2}} & \text { if } k \leq \frac{a_{2}\left(2-a_{2}\right)}{1-a_{2}}, \\
\frac{1}{2-a_{2}+k} & \text { if } k>\frac{a_{2}\left(2-a_{2}\right)}{1-a_{2}},\end{cases} \\
& q_{1} \in\left[0, a_{1}\right] \text { satisfies } k q_{1}<1-\max \left\{q_{2}, \frac{a_{2}-a_{2} q_{2}}{1-2 q_{2}}, \frac{k q_{2}-a_{2} q_{2}}{1-2 q_{2}}\right\} .
\end{aligned}
$$

Here, the latter hypothesis (1.7) itself is equivalent to saying that $k q_{1}+q_{2}<1$ and

$$
\begin{cases}k q_{1}+\left(2-a_{2}\right) q_{2}+a_{2}-2 k q_{1} q_{2}<1 & \text { if } k q_{2}<a_{2} \\ k q_{1}+\left(2-a_{2}+k\right) q_{2}-2 k q_{1} q_{2}<1 & \text { if } k q_{2} \geq a_{2}\end{cases}
$$


Prescribing the above conditions, we obtain the following main result on competitive exclusion.

Theorem 1.1 Assume (1.4), and suppose that $k$ and the numbers $q_{1}$ and $q_{2}$ defined in (1.5) satisfy (1.6). Then for any choice of nonnegative initial data $u_{0} \in C^{0}(\bar{\Omega})$ and $v_{0} \in C^{0}(\bar{\Omega})$ satisfying $v_{0} \not \equiv 0$, the problem $(1.1)-(1.3)$ possesses a uniquely determined global-in-time classical solution $(u, v, w)$ such that $u \geq 0, v>0$ and $w>0$ in $\bar{\Omega} \times(0, \infty)$ and

$$
u(\cdot, t) \rightarrow 0, \quad v(\cdot, t) \rightarrow 1 \quad \text { and } \quad w(\cdot, t) \rightarrow \frac{1}{\lambda} \quad \text { as } t \rightarrow \infty,
$$

uniformly with respect to $x \in \Omega$. Moreover, either $u \equiv 0$ in $\bar{\Omega} \times[0, \infty)$ or $u>0$ in $\bar{\Omega} \times(0, \infty)$ is satisfied.

Let us illustrate how the condition (1.6) becomes easier to handle in some special cases.

Remark (i) In the prototypical case when $\chi_{1}=\chi_{2} \equiv \chi$ and $\mu_{1}=\mu_{2} \equiv \mu,(1.6)$ reduces to the condition that $q:=\frac{\chi}{\mu}$ satisfies $q<\frac{1}{k+1}$ and

$$
q< \begin{cases}\frac{2+k-a_{2}-\sqrt{\left(k+2-a_{2}\right)^{2}-8 k\left(1-a_{2}\right)}}{4 k} & \text { if } a_{2}>k q \\ \frac{2+2 k-a_{2}-\sqrt{\left(2 k+2-a_{2}\right)^{2}-8 k}}{4 k} & \text { if } a_{2} \leq k q .\end{cases}
$$

(ii) If in the above case we moreover have $k=1$ then (1.10) becomes

$$
q< \begin{cases}\frac{4-a_{2}-\sqrt{8-8 a_{2}+a_{2}^{2}}}{4-a_{2}} & \text { if } a_{2} \leq q \\ \frac{1-a_{2}}{2} & \text { if } a_{2}>q .\end{cases}
$$

We observe that the first case can only occur if $a_{2}<\frac{4-a_{2}-\sqrt{8-8 a_{2}+a_{2}^{2}}}{4}$ is satisfied, which is equivalent to $a_{2}<\frac{1}{3}$ in view of $a_{2} \in[0,1)$. Hence, the first case in (1.11) is equivalent to

$$
a_{2}<\frac{1}{3} \text { and } a_{2} \leq q<\frac{4-a_{2}-\sqrt{8-8 a_{2}+a_{2}^{2}}}{4} .
$$

The second case in (1.11) is equivalent to

$$
q<\min \left\{a_{2}, \frac{1-a_{2}}{2}\right\}= \begin{cases}a_{2} & \text { if } a_{2}<\frac{1}{3} \\ \frac{1-a_{2}}{2} & \text { if } a_{2} \in\left[\frac{1}{3}, 1\right)\end{cases}
$$


Combining both cases we conclude

$$
q< \begin{cases}\frac{4-a_{2}-\sqrt{8-8 a_{2}+a_{2}^{2}}}{4} & \text { if } a_{2}<\frac{1}{3} \\ \frac{1-a_{2}}{2} & \text { if } a_{2} \in\left[\frac{1}{3}, 1\right) .\end{cases}
$$

(iii) In the limit case $k=0,(1.10)$ requires that

$$
q<\frac{1-a_{2}}{2-a_{2}}
$$

(iv) Finally, in the borderline case $a_{2}=0,(1.13)$ reads

$$
q<\frac{1}{2}
$$

and is thus consistent with the conditions already found in (Tello and Winkler 2007, Theorem 5.1).

Remark The global existence statement in Theorem 1.1 remains valid if (1.7) is replaced with the weaker requirement that $k q_{1}+q_{2}<1$. In fact, Lemma 2.2 below will show that in this case the interplay of diffusion and kinetics in (1.1) is strong enough to overbalance chemotactic cross-diffusion in such a way that all solutions are global and remain bounded.

The plan of this paper is as follows. In Sect. 2 we show the local existence of a solution along with its positivity properties and prove the existence of a global bounded solution once $k q_{1}+q_{2}<1$ is satisfied. Section 3 contains relations between the possible limits of $u$ and $v$ which are established by using comparison methods in combination with some algebraic inequalities. In particular we show that $v(t) \rightarrow 1$ if $u(t) \rightarrow 0$ is satisfied. In Sect. 4 we then prove that $u$ converges to 0 in the cases $k q_{2}<a_{2}$ and $k q_{2} \geq a_{2}$, respectively, and complete the proof of Theorem 1.1. The final Sect. 5 contains our conclusions and a discussion.

\section{Preliminaries: boundedness}

In this section we state some basic properties of the solutions to (1.1)-(1.3) and give a criterion for their boundedness. We start with the local existence of a solution and its positivity properties.

Lemma 2.1 Suppose that $u_{0}, v_{0} \in C^{0}(\bar{\Omega})$ are nonnegative such that $v_{0} \not \equiv 0$. Then there exists $T_{\max } \in(0, \infty]$ and a unique classical solution $(u, v, w)$ of $(1.1)-(1.3)$ which is nonnegative and belongs to $C^{0}\left(\bar{\Omega} \times\left[0, T_{\max }\right)\right) \cap C^{2,1}\left(\bar{\Omega} \times\left(0, T_{\max }\right)\right)$. Moreover, $v>0$ and $w>0$ in $\bar{\Omega} \times\left(0, T_{\max }\right)$ and either $u \equiv 0$ in $\bar{\Omega} \times\left[0, T_{\max }\right)$ or $u>0$ in $\bar{\Omega} \times\left(0, T_{\text {max }}\right)$ are satisfied. Furthermore, we have the following extensibility criterion:

$$
\text { If } T_{\max }<\infty, \text { then } \limsup _{t \nearrow T_{\max }}\left(\|u(\cdot, t)\|_{L^{\infty}(\Omega)}+\|v(\cdot, t)\|_{L^{\infty}(\Omega)}\right)=\infty \text {. }
$$


Proof The local existence and regularity of the solution as well as the extensibility criterion (2.1) can be proved by a slight adaption of well-known methods. We thus may confine ourselves with an outline of the proof and refer the reader e.g. to Winkler (2010), where details are given in a closely related situation.

For small $T \in(0,1)$, in the space

$$
X:=C^{0}\left([0, T] ; C^{0}(\bar{\Omega})\right) \times C^{0}\left([0, T] ; C^{0}(\bar{\Omega})\right)
$$

we consider the closed set

$S:=\left\{(u, v) \in X \mid\|u\|_{L^{\infty}\left((0, T) ; L^{\infty}(\Omega)\right)} \leq R+1\right.$ and $\left.\|v\|_{L^{\infty}\left((0, T) ; L^{\infty}(\Omega)\right)} \leq R+1\right\}$,

where $R:=\left\|u_{0}+v_{0}\right\|_{L^{\infty}(\Omega)}$. For $(u, v) \in S$, we introduce a mapping $\Phi$ on $S$ by letting $w \in \bigcap_{1<p<\infty} L^{\infty}\left((0, T) ; W^{2, p}(\Omega)\right)$ denote the (weak) solution of

$$
\begin{cases}-\Delta w+\lambda w=k u+v, & x \in \Omega, \\ \frac{\partial w}{\partial v}=0, & x \in \partial \Omega,\end{cases}
$$

and then defining

$$
\begin{aligned}
\Phi(u, v)(t):= & \left(\begin{array}{l}
\Phi_{1}(u, v)(t) \\
\Phi_{2}(u, v)(t)
\end{array}\right) \\
:= & \left(\begin{array}{c}
e^{d_{1} t \Delta} u_{0}+\int_{0}^{t} e^{d_{1}(t-s) \Delta}\left[-\chi_{1} \nabla \cdot(u \nabla w)+f_{1}(u, v)\right](s) d s \\
e^{t} \\
d_{2} t \Delta \\
v_{0}+\int_{0} e^{d_{2}(t-s) \Delta}\left[-\chi_{2} \nabla \cdot(v \nabla w)+f_{2}(u, v)\right](s) d s
\end{array}\right)
\end{aligned}
$$

for $t \in[0, T]$, where $\left(e^{\tau \Delta}\right)_{\tau \geq 0}$ denotes the Neumann heat semigroup, and where

$f_{1}(u, v):=\mu_{1} u\left(1-u-a_{1} v\right)$ and $f_{2}(u, v):=\mu_{2} v\left(1-v-a_{2} u\right), \quad u \in \mathbb{R}, v \in \mathbb{R}$.

Then by a straightforward reasoning involving standard elliptic regularity properties and known smoothing estimates for the heat semigroup (Quittner and Souplet 2007), it is possible to show that if $T=T(R)$ is sufficiently small then $\Phi$ is a contraction on $S$. The accordingly existing fixed point $(u, v)$ of $\Phi$, along with $w$ as gained from (2.2), can then, again by standard regularity arguments, shown to be smooth in $\bar{\Omega} \times(0, T)$ and continuous in $\bar{\Omega} \times[0, T]$ in all its components, and to solve (1.1) classically in $\Omega \times(0, T)$. Since the choice of $T$ depends on $R$ only, (2.1) is now immediate.

An application of the strong maximum principle to the first and second equation of (1.1) implies the claim concerning the positivity of $u$ and $v$. Hence, $k u+v$ is positive in $\bar{\Omega} \times\left(0, T_{\max }\right)$ and the strong elliptic maximum principle applied to the third equation of (1.1) yields positivity also of $w$. 
Finally, taking differences $U:=u_{1}-u_{2}$ and $V:=v_{1}-v_{2}$ of two supposedly existing solutions $\left(u_{i}, v_{i}, w_{i}\right)$ in $\Omega \times(0, T)$ for some $T>0, i \in\{1,2\}$, upon testing the equations for $U$ and $V$ obtained from (1.1) by $U$ and $V$, respectively, in a straightforward manner one can derive an inequality of the form

$$
\frac{1}{2} \frac{d}{d t}\left\{\int_{\Omega} U^{2}+\int_{\Omega} V^{2}\right\} \leq C\left(T^{\prime}\right)\left\{\int_{\Omega} U^{2}+\int_{\Omega} V^{2}\right\} \quad \text { for all } t \in\left(0, T^{\prime}\right)
$$

valid for any fixed $T^{\prime} \in(0, T)$ and some $C\left(T^{\prime}\right)>0$ depending on the bounded quantities $\left\|u_{i}\right\|_{L^{\infty}\left(\Omega \times\left(0, T^{\prime}\right)\right)}$ and $\left\|v_{i}\right\|_{L^{\infty}\left(\Omega \times\left(0, T^{\prime}\right)\right)}, i \in\{1,2\}$. This clearly implies uniqueness.

We now let $\mathcal{L}_{j}=\mathcal{L}_{j}(x, t), j \in\{1,2\}$, the parabolic operators

$$
\mathcal{L}_{j} \varphi:=d_{j} \Delta \varphi-\chi_{j} \nabla w(x, t) \cdot \nabla \varphi, \quad(x, t) \in \Omega \times\left(0, T_{\text {max }}\right),
$$

for $\varphi \in C^{2}(\Omega)$. Then the first and third equation of (1.1) show that

$$
\begin{aligned}
u_{t}-\mathcal{L}_{1} u & =u \cdot\left\{-\chi_{1} \Delta w+\mu_{1}\left(1-u-a_{1} v\right)\right\} \\
& =u \cdot\left\{\mu_{1}-\left(\mu_{1}-k \chi_{1}\right) u-\left(a_{1} \mu_{1}-\chi_{1}\right) v-\lambda \chi_{1} w\right\} \quad \text { in } \Omega \times\left(0, T_{\max }\right) .
\end{aligned}
$$

Similarly, the second and third equation of (1.1) imply

$$
\begin{aligned}
v_{t}-\mathcal{L}_{2} v & =v \cdot\left\{-\chi_{2} \Delta w+\mu_{2}\left(1-v-a_{2} u\right)\right\} \\
& =v \cdot\left\{\mu_{2}-\left(\mu_{2}-\chi_{2}\right) v+\left(k \chi_{2}-a_{2} \mu_{2}\right) u-\lambda \chi_{2} w\right\} \text { in } \Omega \times\left(0, T_{\max }\right) .
\end{aligned}
$$

The final result of this section asserts boundedness of the solution once the ratios $q_{1}$ and $q_{2}$ defined in (1.5) are small enough.

Lemma 2.2 Assume that

$$
k q_{1}+q_{2}<1
$$

Then $T_{\max }=\infty$ and both $u$ and $v$ are bounded in $\Omega \times(0, \infty)$.

Proof According to the fact that $u, v$ and $w$ are all nonnegative by Lemma 2.1, we have

$$
\begin{aligned}
& \mathcal{P}_{1} u:=u_{t}-\mathcal{L}_{1} u-u \cdot\left\{\mu_{1}-\left(\mu_{1}-k \chi_{1}\right) u+\chi_{1} v\right\} \leq 0 \text { in } \Omega \times\left(0, T_{\max }\right) \text { and } \\
& \mathcal{P}_{2} v:=v_{t}-\mathcal{L}_{2} v-v \cdot\left\{\mu_{2}-\left(\mu_{2}-\chi_{2}\right) v+k \chi_{2} u\right\} \leq 0 \text { in } \Omega \times\left(0, T_{\max }\right)
\end{aligned}
$$


where $\mathcal{L}_{1}$ and $\mathcal{L}_{2}$ are defined in (2.3). We now observe that (2.6) is equivalent to

$$
\left(\mu_{1}-k \chi_{1}\right)\left(\mu_{2}-\chi_{2}\right)>k \chi_{1} \chi_{2}
$$

and hence to

$$
\frac{\mu_{1}-k \chi_{1}}{\chi_{1}}>\frac{k \chi_{2}}{\mu_{2}-\chi_{2}}
$$

We can thus pick $\xi>0$ large enough such that

$$
\xi \geq \max \left\{\left\|u_{0}\right\|_{L^{\infty}(\Omega)}, \frac{\mu_{2}-\chi_{2}}{k \chi_{2}}\left\|v_{0}\right\|_{L^{\infty}(\Omega)}\right\}
$$

and that

$$
\frac{\mu_{1}-k \chi_{1}-\frac{\mu_{1}}{\xi}}{\chi_{1}}>\frac{k \chi_{2}+\frac{\mu_{2}}{\xi}}{\mu_{2}-\chi_{2}},
$$

which enables us to find $A>0$ fulfilling

$$
\frac{\mu_{1}-k \chi_{1}-\frac{\mu_{1}}{\xi}}{\chi_{1}}>A>\frac{k \chi_{2}+\frac{\mu_{2}}{\xi}}{\mu_{2}-\chi_{2}} .
$$

Then the constant functions defined by

$$
\bar{u}(x, t):=\xi \text { and } \bar{v}(x, t):=A \xi, \quad(x, t) \in \bar{\Omega} \times\left[0, T_{\max }\right),
$$

satisfy

$$
\bar{u}(x, 0)=\xi \geq u_{0}(x) \text { and } \bar{v}(x, 0)=A \xi>\frac{k \chi_{2}}{\mu_{2}-\chi_{2}} \cdot \xi \geq v_{0}(x) \text { for all } x \in \Omega
$$

by (2.8). Moreover, (2.9) warrants that

$$
\mathcal{P}_{1} \bar{u}=-\xi \cdot\left\{\mu_{1}-\left(\mu_{1}-k \chi_{1}\right) \xi+\chi_{1} \cdot A \xi\right\}>0 \text { in } \Omega \times\left(0, T_{\max }\right)
$$

and

$$
\mathcal{P}_{2} \bar{v}=-A \xi \cdot\left\{\mu_{2}-\left(\mu_{2}-\chi_{2}\right) \cdot A \xi+k \chi_{2} \xi\right\}>0 \text { in } \Omega \times\left(0, T_{\max }\right) .
$$

In view of (2.7) and (2.10), the comparison principle for cooperative reaction-diffusion systems (see for instance Quittner and Souplet 2007, Proposition 52.22) allows us to conclude that $u \leq \bar{u}$ and $v \leq \bar{v}$ in $\Omega \times\left(0, T_{\max }\right)$, which by Lemma 2.1 entails that $T_{\max }=\infty$ and that $u$ and $v$ are globally bounded. 


\section{Some technical inequalities}

According to the above boundedness result, under the assumption (1.6) we know that

$$
\begin{aligned}
& L_{1}:=\limsup _{t \rightarrow \infty}\left(\max _{x \in \bar{\Omega}} u(x, t)\right), \\
& L_{2}:=\limsup _{t \rightarrow \infty}\left(\max _{x \in \bar{\Omega}} v(x, t)\right), \text { and } \\
& l_{2}:=\liminf _{t \rightarrow \infty}\left(\min _{x \in \bar{\Omega}} v(x, t)\right)
\end{aligned}
$$

define finite real numbers satisfying

$$
L_{1} \geq 0 \text { and } 0 \leq l_{2} \leq L_{2}
$$

Proving Theorem 1.1 then amounts to verifying that $L_{1}=0$ and $L_{2}=l_{2}=1$, because the large time behavior of $w$ is then uniquely determined according to the following.

Lemma 3.1 For each $t \in\left(0, T_{\max }\right)$, we have

$$
\min _{y \in \bar{\Omega}} v(y, t) \leq \lambda w(x, t) \leq k \cdot \max _{y \in \bar{\Omega}} u(y, t)+\max _{y \in \bar{\Omega}} v(y, t) \text { for all } x \in \bar{\Omega}
$$

Proof The proof repeats a standard elliptic comparison argument: If $\varphi \in C^{2}(\bar{\Omega})$ denotes an arbitrary function satisfying $\frac{\partial \varphi}{\partial \nu}<0$ on $\partial \Omega$, then for any $\varepsilon>0$, at each point $x_{0} \in \bar{\Omega}$ where $z:=w(\cdot, t)+\varepsilon \varphi$ attains its maximum we necessarily have $x_{0} \in \Omega$ and hence $\Delta z\left(x_{0}\right) \leq 0$. Since $\Delta z=\lambda z-k u-v+\varepsilon(\Delta \varphi-\lambda \varphi)$, this implies that

$$
\begin{aligned}
\lambda z(x) \leq \lambda z\left(x_{0}\right) & \leq k u\left(x_{0}, t\right)+v\left(x_{0}, t\right)-\varepsilon(\Delta \varphi-\lambda \varphi)\left(x_{0}\right) \\
& \leq k \cdot \max _{y \in \bar{\Omega}} u(y, t)+\max _{y \in \bar{\Omega}} v(y, t)+\varepsilon \cdot \max _{y \in \bar{\Omega}}|\Delta \varphi(y)-\lambda \varphi(y)| .
\end{aligned}
$$

Taking $\varepsilon \searrow 0$ we arrive at the right inequality in (3.2), whereas the left can be seen similarly on dropping the nonnegative term $k \cdot \min _{y \in \bar{\Omega}} u(y, t)$.

A first trivial observation linking the asymptotic of $(u, v, w)$ to $L_{1}, L_{2}$ and $l_{2}$ then is the following.

Lemma 3.2 Assume (2.6). Then for all $\varepsilon>0$ there exists $t_{\varepsilon}>0$ such that

$$
u(x, t) \leq L_{1}+\varepsilon \text { for all } x \in \bar{\Omega} \text { and } t \geq t_{\varepsilon},
$$

that

$$
l_{2}-\varepsilon \leq v(x, t) \leq L_{2}+\varepsilon \text { for all } x \in \bar{\Omega} \text { and } t \geq t_{\varepsilon},
$$


and that

$$
l_{2}-\varepsilon \leq \lambda w(x, t) \leq k\left(L_{1}+\varepsilon\right)+\left(L_{2}+\varepsilon\right) \text { for all } x \in \bar{\Omega} \text { and } t \geq t_{\varepsilon}
$$

Proof That (3.3) and (3.4) can be achieved for suitably large $t_{\varepsilon}$ is an immediate consequence of the definitions in (3.1). Then applying Lemma 3.1 for fixed $t \geq t_{\varepsilon}$ we readily obtain $(3.5)$.

Next we compare $u$ with a suitable spatially homogeneous function and obtain an upper bound for $L_{1}$ in terms of $l_{2}$.

Lemma 3.3 Assume (1.6). Then the numbers $L_{1}$ and $l_{2}$ defined in (3.1) fulfill the relation

$$
\left(1-k q_{1}\right) L_{1} \leq\left(1-a_{1} l_{2}\right)_{+}
$$

Proof If $u \equiv 0$ in $\bar{\Omega} \times[0, \infty)$, then (3.6) is fulfilled in view of $L_{1}=0$. Otherwise, according to (2.4), taking $\mathcal{L}_{1}$ as in (2.3) we recall that

$$
u_{t}=\mathcal{L}_{1} u+u \cdot\left\{\mu_{1}-\left(\mu_{1}-k \chi_{1}\right) u-\left(a_{1} \mu_{1}-\chi_{1}\right) v-\lambda \chi_{1} w\right\} \text { in } \Omega \times(0, \infty),
$$

where (1.6) ensures that $a_{1} \mu_{1}-\chi_{1} \geq 0$. Thus, if for fixed $\varepsilon>0$ we take $t_{\varepsilon}$ as given by Lemma 3.2, then (3.4) and (3.5) yield

$$
-\left(a_{1} \mu_{1}-\chi_{1}\right) v \leq-\left(a_{1} \mu_{1}-\chi_{1}\right) \cdot\left(l_{2}-\varepsilon\right) \text { in } \Omega \times\left(t_{\varepsilon}, \infty\right)
$$

and

$$
-\lambda \chi_{1} w \leq-\chi_{1} \cdot\left(l_{2}-\varepsilon\right) \text { in } \Omega \times\left(t_{\varepsilon}, \infty\right) \text {, }
$$

and therefore we obtain

$$
u_{t} \leq \mathcal{L}_{1} u+u \cdot\left\{\mu_{1}-\left(\mu_{1}-k \chi_{1}\right) u-a_{1} \mu_{1}\left(l_{2}-\varepsilon\right)\right\} \quad \text { in } \Omega \times\left(t_{\varepsilon}, \infty\right)
$$

Since $\mathcal{L}_{1}$ annihilates spatially homogeneous functions, a parabolic comparison argument hence implies that

$$
u(x, t) \leq \bar{u}(t) \text { for all } x \in \bar{\Omega} \text { and } t \geq t_{\varepsilon},
$$

where $\bar{u}$ denotes the solution of the initial-value problem

$$
\left\{\begin{array}{l}
\bar{u}^{\prime}=\bar{u} \cdot\left\{\mu_{1}-\left(\mu_{1}-k \chi_{1}\right) \bar{u}-a_{1} \mu_{1}\left(l_{2}-\varepsilon\right)\right\}, \quad t>t_{\varepsilon}, \\
\bar{u}\left(t_{\varepsilon}\right)=\max _{x \in \bar{\Omega}} u\left(x, t_{\varepsilon}\right) .
\end{array}\right.
$$


Since $u\left(\cdot, t_{\varepsilon}\right)$ is positive in $\bar{\Omega}$ by Lemma 2.1 , it is clear that

$$
\bar{u}(t) \rightarrow \max \left\{0, \frac{\mu_{1}-a_{1} \mu_{1}\left(l_{2}-\varepsilon\right)}{\mu_{1}-k \chi_{1}}\right\} \quad \text { as } t \rightarrow \infty,
$$

which in conjunction with (3.7) yields the inequality

$$
\limsup _{t \rightarrow \infty}\left(\max _{x \in \bar{\Omega}} u(x, t)\right) \leq \max \left\{0, \frac{\mu_{1}-a_{1} \mu_{1}\left(l_{2}-\varepsilon\right)}{\mu_{1}-k \chi_{1}}\right\} .
$$

Taking $\varepsilon \searrow 0$ now shows that indeed (3.6) must be valid.

In order to study the large time behavior of $v$ we need to distinguish two cases depending on the sign of $k q_{2}-a_{2}$. We again use comparison arguments involving spatially homogeneous functions and first give the result for $k q_{2}<a_{2}$.

Lemma 3.4 Suppose that (1.6) holds, and that $k q_{2}<a_{2}$. Then

$$
\left(1-q_{2}\right) L_{2} \leq\left(1-q_{2} l_{2}\right)_{+}
$$

and

$$
\left(1-q_{2}\right) l_{2} \geq 1-a_{2} L_{1}-q_{2} L_{2}
$$

Proof The procedure is similar to that in Lemma 3.3: Given $\varepsilon>0$, we take $t_{\varepsilon}>0$ as provided by Lemma 3.2. We recall that by (2.5) we have

$$
v_{t}=\mathcal{L}_{2} v+v \cdot\left\{\mu_{2}-\left(\mu_{2}-\chi_{2}\right) v-\left(a_{2} \mu_{2}-k \chi_{2}\right) u-\lambda \chi_{2} w\right\} \quad \text { in } \Omega \times(0, \infty)
$$

with $\mathcal{L}_{2}$ given by (2.3). Since $a_{2} \mu_{2}-k \chi_{2}$ is nonnegative according to our hypothesis $k q_{2}<a_{2}$, using that $u \geq 0$ we can estimate

$$
-\left(a_{2} \mu_{2}-k \chi_{2}\right) u \leq 0 \text { in } \Omega \times(0, \infty)
$$

whereas by (3.5),

$$
-\lambda \chi_{2} w \leq-\chi_{2} \cdot\left(l_{2}-\varepsilon\right) \text { in } \Omega \times\left(t_{\varepsilon}, \infty\right) .
$$

Thus, (3.10) implies that

$$
v_{t} \leq \mathcal{L}_{2} v+v \cdot\left\{\mu_{2}-\left(\mu_{2}-\chi_{2}\right) v-\chi_{2}\left(l_{2}-\varepsilon\right)\right\} \quad \text { in } \Omega \times\left(t_{\varepsilon}, \infty\right),
$$

whence by comparison we find that

$$
v(x, t) \leq \bar{v}(t) \text { for all } x \in \bar{\Omega} \text { and } t \geq t_{\varepsilon},
$$


if we let $\bar{v}$ denote the solution of

$$
\left\{\begin{array}{l}
\bar{v}^{\prime}=\bar{v} \cdot\left\{\mu_{2}-\left(\mu_{2}-\chi_{2}\right) \bar{v}-\chi_{2}\left(l_{2}-\varepsilon\right)\right\}, \quad t>t_{\varepsilon} \\
\bar{v}\left(t_{\varepsilon}\right)=\max _{x \in \bar{\Omega}} v\left(x, t_{\varepsilon}\right)
\end{array}\right.
$$

In light of the long time asymptotics of $\bar{v}$, this entails that

$$
\limsup _{t \rightarrow \infty}\left(\max _{x \in \bar{\Omega}} v(x, t)\right) \leq \max \left\{0, \frac{\mu_{2}-\chi_{2}\left(l_{2}-\varepsilon\right)}{\mu_{2}-\chi_{2}}\right\}
$$

for any $\varepsilon>0$ and hence

$$
L_{2} \leq \max \left\{0, \frac{\mu_{2}-\chi_{2} l_{2}}{\mu_{2}-\chi_{2}}\right\}
$$

which proves (3.8).

Similarly, (3.9) can be obtained by going back to (3.10) and using (3.3) and (3.5) to estimate

$$
-\left(a_{2} \mu_{2}-k \chi_{2}\right) u \geq-\left(a_{2} \mu_{2}-k \chi_{2}\right) \cdot\left(L_{1}+\varepsilon\right) \text { in } \Omega \times\left(t_{\varepsilon}, \infty\right)
$$

and

$$
-\lambda \chi_{2} w \geq-\chi_{2} \cdot\left(k L_{1}+L_{2}+(k+1) \varepsilon\right) \text { in } \Omega \times\left(t_{\varepsilon}, \infty\right),
$$

again because $k \chi_{2} \leq a_{2} \mu_{2}$. We thereupon obtain

$$
\begin{aligned}
v_{t}-\mathcal{L}_{2} v \geq & v \cdot\left\{\mu_{2}-\left(\mu_{2}-\chi_{2}\right) v-\left(a_{2} \mu_{2}-k \chi_{2}\right)\right. \\
& \left.\cdot\left(L_{1}+\varepsilon\right)-\chi_{2}\left(k L_{1}+L_{2}+(k+1) \varepsilon\right)\right\} \\
= & v \cdot\left\{\mu_{2}-\left(\mu_{2}-\chi_{2}\right) v-a_{2} \mu_{2} \cdot\left(L_{1}+\varepsilon\right)-\chi_{2}\left(L_{2}+\varepsilon\right)\right\} \\
& \quad \text { in } \Omega \times\left(t_{\varepsilon}, \infty\right),
\end{aligned}
$$

whence

$$
v(x, t) \geq \underline{v}(t) \text { for all } x \in \bar{\Omega} \text { and } t \geq t_{\varepsilon},
$$

by the comparison principle, where

$$
\left\{\begin{array}{l}
\underline{v}^{\prime}=\underline{v} \cdot\left\{\mu_{2}-\left(\mu_{2}-\chi_{2}\right) \underline{v}-a_{2} \mu_{2}\left(L_{1}+\varepsilon\right)-\chi_{2}\left(L_{2}+\varepsilon\right)\right\}, \quad t>t_{\varepsilon} \\
\underline{v}\left(t_{\varepsilon}\right)=\min _{x \in \Omega} v\left(x, t_{\varepsilon}\right)
\end{array}\right.
$$

Now an important observation, singling out the particular steady state solution $(\tilde{u}, \tilde{v}, \tilde{w}) \equiv\left(1,0, \frac{1}{\lambda}\right)$ for which (3.9) does not hold, is that $\underline{v}\left(t_{\varepsilon}\right)$ is positive thanks 
to the positivity of $v$ in $\bar{\Omega} \times(0, \infty)$ asserted by Lemma 2.1 . Consequently, $\underline{v}$ again approaches the larger of the equilibria of (3.12), that is, we have

$$
\underline{v}(t) \rightarrow \max \left\{0, \frac{\mu_{2}-a_{2} \mu_{2}\left(L_{1}+\varepsilon\right)-\chi_{2}\left(L_{2}+\varepsilon\right)}{\mu_{2}-\chi_{2}}\right\} \quad \text { as } t \rightarrow \infty,
$$

which in the limit $\varepsilon \searrow 0$ clearly implies (3.9).

In case of $k q_{2} \geq a_{2}$ we proceed in a similar way.

Lemma 3.5 Assume (1.6), and suppose that $k q_{2} \geq a_{2}$. Then

$$
\left(1-q_{2}\right) L_{2} \leq\left(1+\left(k q_{2}-a_{2}\right) L_{1}-q_{2} l_{2}\right)_{+}
$$

and

$$
\left(1-q_{2}\right) l_{2} \geq 1-k q_{2} L_{1}-q_{2} L_{2}
$$

Proof Again using (2.5) as a starting point, given $\varepsilon>0$ we take $t_{\varepsilon}>0$ as given by Lemma 3.2 and estimate

$$
\left(k \chi_{2}-a_{2} \mu_{2}\right) u \leq\left(k \chi_{2}-a_{2} \mu_{2}\right) \cdot\left(L_{1}+\varepsilon\right) \text { in } \Omega \times\left(t_{\varepsilon}, \infty\right)
$$

and

$$
-\lambda \chi_{2} w \leq-\chi_{2} \cdot\left(l_{2}-\varepsilon\right) \text { in } \Omega \times\left(t_{\varepsilon}, \infty\right) \text {. }
$$

We thereupon obtain from the identity

$$
v_{t}=\mathcal{L}_{2} v+v \cdot\left\{\mu_{2}-\left(\mu_{2}-\chi_{2}\right) v+\left(k \chi_{2}-a_{2} \mu_{2}\right) u-\lambda \chi_{2} w\right\} \text { in } \Omega \times(0, \infty),
$$

as obtained in (2.5), that

$$
\begin{aligned}
v_{t} \leq & \mathcal{L}_{2} v+v \cdot\left\{\mu_{2}-\left(\mu_{2}-\chi_{2}\right) v+\left(k \chi_{2}-a_{2} \mu_{2}\right)\left(L_{1}+\varepsilon\right)-\chi_{2}\left(l_{2}-\varepsilon\right)\right\} \\
& \text { in } \Omega \times\left(t_{\varepsilon}, \infty\right) .
\end{aligned}
$$

By comparison with spatially homogeneous ODE solutions in the same manner as in Lemma 3.4, we thereby derive the inequality

$$
\limsup _{t \rightarrow \infty}\left(\max _{x \in \bar{\Omega}} v(x, t)\right) \leq \max \left\{0, \frac{\mu_{2}+\left(k \chi_{2}-a_{2} \mu_{2}\right)\left(L_{1}+\varepsilon\right)-\chi_{2}\left(l_{2}-\varepsilon\right)}{\mu_{2}-\chi_{2}}\right\},
$$

which on taking $\varepsilon \searrow 0$ yields (3.13).

Similarly, inserting the lower estimates

$$
\left(k \chi_{2}-a_{2} \mu_{2}\right) u \geq 0 \text { in } \Omega \times(0, \infty)
$$


and

$$
-\lambda \chi_{2} w \geq-\chi_{2} \cdot\left(k L_{1}+L_{2}+(k+1) \varepsilon\right) \text { in } \Omega \times\left(t_{\varepsilon}, \infty\right)
$$

into (3.15) shows that

$$
v_{t} \geq \mathcal{L}_{2} v+v \cdot\left\{\mu_{2}-\left(\mu_{2}-\chi_{2}\right) v-\chi_{2}\left(k L_{1}+L_{2}+(k+1) \varepsilon\right)\right\} \text { in } \Omega \times\left(t_{\varepsilon}, \infty\right),
$$

which on comparison entails that

$$
\liminf _{t \rightarrow \infty}\left(\min _{x \in \bar{\Omega}} v(x, t)\right) \geq \frac{\mu_{2}-\chi_{2}\left(k L_{1}+L_{2}+(k+1) \varepsilon\right)}{\mu_{2}-\chi_{2}}
$$

and thereby proves (3.14).

Using the estimates shown in this section, we are now able to prove that $v(t) \rightarrow 1$ in $L^{\infty}(\Omega)$ as $t \rightarrow \infty$ if we assume $L_{1}=0$.

Lemma 3.6 Assume that (1.6) holds, and suppose that $L_{1}=0$. Then $L_{2}=l_{2}=1$.

Proof We first observe that $q_{2} l_{2} \leq 1$, for otherwise by either (3.8) or by (3.13) in combination with $L_{1}=0$ we would have $L_{2}=0$ and hence could draw the conclusion that $l_{2}=0$ which is absurd in view of (3.9) and (3.14).

Accordingly, in light of the hypothesis $L_{1}=0$, Lemma 3.4 and Lemma 3.5 show that in both cases $k q_{2}<a_{2}$ and $k q_{2} \geq a_{2}$, the inequalities

$$
\left(1-q_{2}\right) L_{2} \leq 1-q_{2} l_{2}
$$

and

$$
\left(1-q_{2}\right) l_{2} \geq 1-q_{2} L_{2}
$$

hold, which on subtraction imply

$$
\left(1-q_{2}\right)\left(L_{2}-l_{2}\right) \leq q_{2}\left(L_{2}-l_{2}\right)
$$

Since (1.6) implies that $q_{2}<\frac{1}{2}$, this asserts that $L_{2} \leq l_{2}$ and hence $L_{2}=l_{2}$. Therefore, once more applying (3.16) shows that $L_{2} \leq 1$, while similarly (3.17) entails that $l_{2} \geq 1$. This completes the proof.

\section{Asymptotic behavior}

According to Lemmas 2.1, 2.2, 3.2 and 3.6, in order to prove Theorem 1.1 it remains to show that $L_{1}=0$ is indeed valid. This will be done by considering again two cases depending on the sign of $k q_{2}-a_{2}$. 
4.1 The case $k q_{2}<a_{2}$

Combining Lemmas 3.3 and 3.4, we complete the proof of Theorem 1.1 for $k q_{2}<a_{2}$.

Lemma 4.1 Suppose that (1.6) holds as well as $k q_{2}<a_{2}$. Then $L_{1}=0$.

Proof Let us suppose on the contrary that $L_{1}$ be positive. Then Lemma 3.3 says that

$$
\left(1-k q_{1}\right) L_{1} \leq 1-a_{1} l_{2}
$$

and hence

$$
l_{2}<\frac{1}{a_{1}}
$$

Here we observe that by (4.2) we also have $1-q_{2} l_{2}>1-\frac{q_{2}}{a_{1}} \geq 1-\frac{1}{a_{1}}>0$ due to the fact that (1.6) entails that $q_{2} \leq 1$. Consequently, Lemma 3.4 asserts that

$$
\left(1-q_{2}\right) L_{2} \leq 1-q_{2} l_{2}
$$

and

$$
\left(1-q_{2}\right) l_{2} \geq 1-a_{2} L_{1}-q_{2} L_{2}
$$

Now combining (4.4) with (4.1) yields

$$
\begin{aligned}
q_{2} L_{2} & \geq 1-a_{2} L_{1}-\left(1-q_{2}\right) l_{2} \\
& \geq 1-a_{2} \cdot \frac{1-a_{1} l_{2}}{1-k q_{1}}-\left(1-q_{2}\right) l_{2} \\
& =1-\frac{a_{2}}{1-k q_{1}}+\left(\frac{a_{1} a_{2}}{1-k q_{1}}-1+q_{2}\right) \cdot l_{2}
\end{aligned}
$$

which in light of (4.3) shows that

$$
\frac{1-q_{2}}{q_{2}} \cdot\left\{1-\frac{a_{2}}{1-k q_{1}}+\left(\frac{a_{1} a_{2}}{1-k q_{1}}-1+q_{2}\right) \cdot l_{2}\right\} \leq\left(1-q_{2}\right) L_{2} \leq 1-q_{2} l_{2} \text {. }
$$

Thus, necessarily

$$
\frac{1-q_{2}}{q_{2}} \cdot\left(1-\frac{a_{2}}{1-k q_{1}}\right)-1 \leq\left\{-\frac{1-q_{2}}{q_{2}} \cdot\left(\frac{a_{1} a_{2}}{1-k q_{1}}-1+q_{2}\right)-q_{2}\right\} \cdot l_{2}
$$

which on multiplication by $\left(1-k q_{1}\right) q_{2}$ can be seen to be equivalent to

$$
\begin{aligned}
& \left\{1-a_{1} a_{2}-k q_{1}-\left(2-a_{1} a_{2}\right) q_{2}+2 k q_{1} q_{2}\right\} \cdot l_{2} \geq 1-a_{2} \\
& \quad-k q_{1}-\left(2-a_{2}\right) q_{2}+2 k q_{1} q_{2} .
\end{aligned}
$$


Since according to (1.8),

$$
I:=1-a_{2}-k q_{1}-\left(2-a_{2}\right) q_{2}+2 k q_{1} q_{2}
$$

is positive, (4.5) is thus only possible if also

$$
J:=1-a_{1} a_{2}-k q_{1}-\left(2-a_{1} a_{2}\right) q_{2}+2 k q_{1} q_{2}
$$

is positive. Therefore, (4.5) implies that

$$
l_{2} \geq \frac{I}{J}
$$

which in conjunction with (4.2) says that $a_{1} I<J$, that is,

$$
\begin{gathered}
a_{1}-a_{1} a_{2}-a_{1} k q_{1}-a_{1}\left(2-a_{2}\right) q_{2}+2 a_{1} k q_{1} q_{2}<1 \\
-a_{1} a_{2}-k q_{1}-\left(2-a_{1} a_{2}\right) q_{2}+2 k q_{1} q_{2} .
\end{gathered}
$$

A simple rearrangement thus yields

$$
\left(a_{1}-1\right) \cdot\left(1-k q_{1}-2 q_{2}+2 k q_{1} q_{2}\right)<0,
$$

which is incompatible with the assumption $I>0$, because $a_{1}>1$ and

$$
1-k q_{1}-2 q_{2}+2 k q_{1} q_{2}=I+a_{2}\left(1-q_{2}\right) \geq I>0
$$

thanks to the fact that $q_{2}<1$ by (1.6). This contradiction shows that actually $L_{1}$ must vanish.

\subsection{The case $k q_{2} \geq a_{2}$}

Finally, a combination of Lemmas 3.3 and 3.5 completes the proof of Theorem 1.1 also for $k q_{2} \geq a_{2}$ like in the preceding section. The details of the proof are given in the following Lemma.

Lemma 4.2 Let (1.6) hold, and assume that $k q_{2} \geq a_{2}$. Then $L_{1}=0$.

Proof If $L_{1}$ was positive, again Lemma 3.3 would yield

$$
l_{2}<\frac{1}{a_{1}}
$$

and

$$
L_{1} \leq \frac{1-a_{1} l_{2}}{1-k q_{1}}
$$


On the other hand, since (4.6) and $k q_{2} \geq a_{2}$ imply $1+\left(k q_{2}-a_{2}\right) L_{1}-q_{2} l_{2}>$ $1-\frac{q_{2}}{a_{1}} \geq 1-\frac{1}{a_{1}}>0$, Lemma 3.5 says that

$$
\left(1-q_{2}\right) L_{2} \leq 1+\left(k q_{2}-a_{2}\right) L_{1}-q_{2} l_{2},
$$

which combined with (4.7) implies

$$
\begin{aligned}
\left(1-q_{2}\right) L_{2} & \leq 1+\left(k q_{2}-a_{2}\right) \cdot \frac{1-a_{1} l_{2}}{1-k q_{1}}-q_{2} l_{2} \\
& =\left(1+\frac{k q_{2}-a_{2}}{1-k q_{1}}\right)-\left\{\frac{a_{1}\left(k q_{2}-a_{2}\right)}{1-k q_{1}}+q_{2}\right\} \cdot l_{2},
\end{aligned}
$$

because $k q_{2} \geq a_{2}$. Moreover, the second statement in Lemma 3.5 asserts that

$$
\left(1-q_{2}\right) l_{2} \geq 1-k q_{2} L_{1}-q_{2} L_{2}
$$

which in view of (4.7) and (4.9) becomes

$$
\begin{aligned}
\left(1-q_{2}\right) l_{2} \geq & 1-k q_{2} \cdot \frac{1-a_{1} l_{2}}{1-k q_{1}}-\frac{q_{2}}{1-q_{2}} \cdot\left(1+\frac{k q_{2}-a_{2}}{1-k q_{1}}\right) \\
& +\frac{q_{2}}{1-q_{2}} \cdot\left\{\frac{a_{1}\left(k q_{2}-a_{2}\right)}{1-k q_{1}}+q_{2}\right\} \cdot l_{2} .
\end{aligned}
$$

When multiplied by $\left(1-k q_{1}\right)\left(1-q_{2}\right)$, this yields

$$
\begin{aligned}
& \left\{\left(1-k q_{1}\right)\left(1-q_{2}\right)^{2}-a_{1} k q_{2}\left(1-q_{2}\right)-a_{1} q_{2}\left(k q_{2}-a_{2}\right)-q_{2}^{2}\left(1-k q_{1}\right)\right\} \cdot l_{2} \\
& \geq\left(1-k q_{1}\right)\left(1-q_{2}\right)-k q_{2}\left(1-q_{2}\right)-\left(1-k q_{1}\right) q_{2}-q_{2}\left(k q_{2}-a_{2}\right),
\end{aligned}
$$

which can be simplified so as to become

$$
J \cdot l_{2} \geq I
$$

where

$$
I:=1-k q_{1}-\left(2+k-a_{2}\right) q_{2}+2 k q_{1} q_{2}
$$

is positive thanks to (1.8), and hence also

$$
J:=1-k q_{1}-\left(2-a_{1} a_{2}+k a_{1}\right) q_{2}+2 k q_{1} q_{2}
$$

must be positive. We thus have $l_{2} \geq \frac{I}{J}$, whence we may conclude using (4.6) that $a_{1} I<J$, that is,

$$
\begin{aligned}
& a_{1}-a_{1} k q_{1}-\left(2 a_{1}+k a_{1}-a_{1} a_{2}\right) q_{2}+2 a_{1} k q_{1} q_{2}<1-k q_{1} \\
& -\left(2-a_{1} a_{2}+k a_{1}\right) q_{2}+2 k q_{1} q_{2} .
\end{aligned}
$$


However, this is equivalent to

$$
\left(a_{1}-1\right)\left(1-k q_{1}\right)\left(1-2 q_{2}\right)<0,
$$

which contradicts (1.6), because clearly $k q_{1}<1$ and $q_{2}<\frac{1}{2}$.

\section{Conclusions and discussion}

In this paper we have considered two biological species which compete for the same resources and migrate chemotactically towards a higher concentration of a chemical substance, which they produce. The problem is modeled by using a system of three partial differential equations: two nonlinear parabolic equations to describe the evolution of the biological species and a linear PDE to model the behavior of the chemical. This chemical diffuses considerably faster than the living organism, and it is thus assumed that the evolution of the chemical signal is governed by an elliptic equation.

The system contains several parameters which measure different aspects in the system: chemotaxis effects, competition, diffusion, chemical production and decay. In the case when competition is absent, it is known that due to chemotaxis, the considered system may produce finite-time blow-up (Espejo et al. 2010), while if on the other hand chemotactic effects are blinded out, then the competitive terms keep the solution bounded and guarantee their global existence. A natural and challenging question has been posed in the literature for such systems: Which are the constraints and the threshold values that decide between driving the system toward global existence, or enforcing blow-up? This question remains open even in the case of a single species. In that case the competitive term is simplified to a logistic growth function (cf. Tello and Winkler 2007 for partial results).

A second question concerns the influence of chemotaxis effects on the stability of the homogeneous steady states determined by the competitive terms. The presence of a large number of parameters in the system makes this question difficult to answer. In the case where the competitive terms are weak in the sense that in (1.1) we have $a_{i} \in[0,1)$ for $i=1,2$, a partial answer is given in Tello and Winkler (2012) within some range of the chemotactic parameters. In this paper we have studied the problem under the assumption that when compared to the latter setting, one of the species is significantly more aggressive in terms of competition.

In this framework, characterized by the assumption (1.4), we have seen that if in (1.5), both ratios $q_{i}, i=1,2$, between the chemotactic sensitivities $\chi_{i}$ and the competition parameters $\mu_{i}$ are suitably small then all nontrivial solutions will be global in time and bounded, and that they approach the homogeneous steady state in which the aggressive subpopulation is at its carrying capacity and the less aggressive species has died out. This inter alia shows that the phenomenon of (asymptotic) extinction of one species, known to be valid for the associated Lotka-Volterra ODE system without diffusion and chemotaxis, persists also in such systems with chemotactic interaction, provided the latter is sufficiently weak. Global existence of solutions is obtained under the assumption $q_{1} k+q_{2}<1$. In that case competition prevents blow-up but extra assumptions are required to prove the stability claim in (1.9). 
We do not know in how far the set (1.6) of hypotheses under which our results have been derived is optimal. After all, in some known borderline cases our approach yields requirements which are consistent with assumptions made in the literature for correspondingly simplified models (cf. the discussion in the remark following Theorem 1.1). In light of results from the literature on corresponding single-species systems, it seems natural to conjecture that for suitably large values of $q_{i}$, solutions may exhibit more colorful dynamics. Indeed, in such a setting numerical simulations indicate that chaotic behavior may occur (Painter and Hillen 2011). It is conceivable that some solutions may even blow up in finite time, but a substantial influence of the space dimension $n$ on the occurrence of such explosion phenomena is most likely to be expected: In the single-species case, for instance, although some examples of high-dimensional blow-up phenomena despite logistic-type growth restrictions have been found for $n \geq 5$ (Winkler 2011), it is known that blow-up never occurs when $n \leq 2$ (Tello and Winkler 2007). In particular, the detection of explosions must thus be restricted to the case $n \geq 3$ in which even numerical approaches seem delicate. As opposed to this, our assumptions in this paper are completely independent of $n$, and moreover they are fully explicit; thereby our results reveal, in a quantitative manner, a stability feature of the competitive exclusion phenomenon with respect to chemotactic interaction.

Acknowledgments The second author is partially supported by Ministerio de Economía y Competitividad under grant MTM2009-13655 (Spain) and CCG07-UPM/000-3199 at UPM.

\section{References}

Brencic A, Winans SC (2005) Detection of and response to signals involved in host-microbe interactions by plant-associated bacteria. Microbiol Mol Biol Rev 69:155-194

Celani A, Vergassola M (2010) Bacterial strategies for chemotaxis response. Proc Natl Acad Sci 107(4):1391-1396

Dung L (2000) Coexistence with chemotaxis. SIAM J Math Anal 32:504-521

Dung L, Smith HL (1999) Steady states of models of microbial growth and competition with chemotaxis. J Math Anal Appl 229:295-318

Espejo EE, Stevens A, Velázquez JJL (2010) A note on non-simultaneous blow-up for a drift-diffusion model. Differ Integral Equ 23(5-6):451-462

Hawkins JB, Jones MT, Plassmann PE, Thorley-Lorson DE (2011) Chemotaxis in densely populated tissue determines germinal center anatomy and cell motility: a new paradigm for the development of complex tissues. PLOS ONE 6(12): 27650

Hibbing ME, Fuqua C, Parsek MR, Peterson SB (2010) Bacterial competition: surviving and thriving in the microbial jungle. Nat Rev Microbiol 8(1):15-25

Kelly FX, Dapsis KJ, Lauffenburger DA (1988) Effect of bacterial chemotaxis on dynamics of microbial competition. Microb Ecol 16:115-131

Kuiper HJ (2001) A priori bounds and global existence for a strongly coupled parabolic system modeling chemotaxis. Electron J Differ Equ 2001(52):1-18

Lauffenburger DA (1991) Quantitative studies of bacterial chemotaxis and microbial population dynamics. Microb Ecol 22:175-185

Murray JD (1993) Mathematical Biology, 2nd edn. Biomathematics series, vol. 19. Springer, Berlin

Painter KJ, Hillen T (2011) Spatio-temporal chaos in a chemotaxis model. Physica D 240:363-375

Painter KJ, Sherratt JA (2003) Modelling the movement of interacting cell populations. J Theor Biol 225:327-339

Quittner P, Souplet P (2007) Superlinear parabolic problems: blow-up, global existence and steady states. Birkhäuser advanced texts. Birkhäuser Verlag, Basel 
Tello JI, Winkler M (2007) A chemotaxis system with logistic source. Commun Partial Differ Equ 32(6):849-877

Tello JI, Winkler M (2012) Stabilization in a two-species chemotaxis system with a logistic source. Nonlinearity $25: 1413-1425$

Tindall MJ, Maini PK, Porter SL, Armitage JP (2008) Overview of mathematical approaches used to model bacterial chemotaxis II: bacterial populations. Bull Math Biol 70:1570-1607

Vande Broek A, Vanderleyden J (1995) The role of bacterial motility, chemotaxis, and attachment in bacteria-plant interactions. Mol Plant Microbe Interact 8:800-810

Wang XF, Wu YP (2002) Qualitative analysis on a chemotactic diffusion model for two species competing for a limited resource. Q Appl Math 60:505-531

Winkler M (2010) Boundedness in the higher-dimensional parabolic-parabolic chemotaxis system with logistic source. Commun Partial Differ Equ 35:1516-1537

Winkler M (2011) Blow-up in a higher-dimensional chemotaxis system despite logistic growth restriction. J Math Anal Appl 384:261-272

Yao J, Allen C (2006) Chemotaxis is required for virulence and competitive fitness of the bacterial wilt pathogen Ralstonia solanacearum. J Bacteriol 188:3697-3708

Zeeman ML (1995) Extinction in competitive Lotka-Volterra systems. Proc AMS 123:87-96

Zhang Z (2006) Existence of global solution and nontrivial steady states for a system modeling chemotaxis. Abstr Appl Anal 2006:1-23. Article ID 81265 